\title{
Multicentric castleman disease in the uncontrolled diabetic
}

\author{
Jessica Y Chambers MD*
}

Department of Internal Medicine, University of Texas, Austin Dell, USA

Castleman Disease is a lymphoproliferative disorder with a diverse array of well-documented associations, most notably human immunodeficiency virus (HIV) and human herpesvirus 8 (HHV-8). It may present as either unicentric or multicentric distribution of disease. Although rare, the mortality from untreated multicentric disease is high, especially in those with autoimmune dysregulation. The disease is often caught late in its progression.

A 33 year old male with uncontrolled type II diabetes was admitted to the hospital one evening for an asthma exacerbation. While his respiratory status resolved quickly with proper treatment, he asks why he has so many "lumps" all over his body. On questioning he admits he has lost 75 pounds in the past few months without trying, complains of significant night sweats, and notes he has not been treating his diabetes appropriately for over a year due to treatment fatigue. On exam he is cachectic, diaphoretic, and tachycardic with nontender lymphadenopathy of the anterior and posterior cervical chains, as well as the axillary, epitrochlear, and femoral regions bilaterally. Laboratory studies were notable for a moderately elevated ESR and LDH, as well as a fasting blood glucose level of over $600 \mathrm{mg} / \mathrm{dL}$ without ketones in the serum or urine. A1C level was greater than 14 . HIV testing was negative. Serum protein electrophoresis showed a faint monoclonal protein in the alpha-2 region and a polyclonal increase in gamma globulins. Imaging revealed diffusely prominent intrathoracic and inguinal lymph nodes over 1 centimeter in diameter. Excisional biopsy of the left inguinal lymph node exhibited follicles with frequently atretic germinal centers containing prominent hyalinized vessels 1 . Immunohistochemistry was negative for human herpesvirus 8 (HHV-8). Interleukin-6 (IL-6) levels by multiplex bead assay were undetectable (Figure 1).

The patient was in the hospital for one week, during which time his glucose was aggressively controlled. His lymphadenopathy objectively

Copyright: (C)2019 Chambers JY. This is an open-access article distributed under the terms of the Creative Commons Attribution License, which permits unrestricted use, distribution, and reproduction in any medium, provided the original author and source are credited.

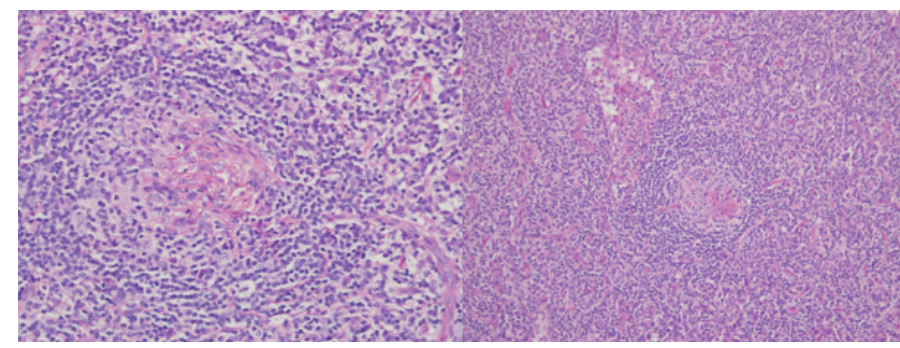

Figure 1. Pathology demonstrating typical features of Castleman Disease with hyalinized vessels and lymphoproliferative changes

decreased in size and his fatigue was resolved. At the time he was seen in primary care clinic weeks later, he had self-discontinued all of his oral diabetic medications and insulin. His disease appeared to have progressed, and his malaise had worsened. He reluctantly agreed to restart his medication regimen at a less aggressive level. He is currently undergoing rituximab infusions under the direction of hematology-oncology.

The salient point in this case is the onset of multicentric lymphoproliferative disease in the setting of significant glucose toxicity. Multicentric Castleman disease also has a well-described association with another constellation of symptoms called POEMS syndrome (Polyneuropathy, Organomegaly, Endocrinopathy, Monoclonal gammopathy, and Skin or bone changes). It is probable this patient's presentation is a variant of POEMS syndrome, with endocrinopathy manifesting as severe insulin resistance. Even more compelling is the evidence that glucose control may prevent the progression of disease in this patient. This adds to the growing body of literature suggesting autoimmune dysregulation as an important, and possibly unrecognized, complication of uncontrolled diabetes.
*Correspondence to: Jessica Y Chambers $\mathrm{MD}^{\star}$, Department of Internal Medicine, University of Texas, Austin Dell, USA, Tel: (713) 376-2524; E-mail: jessica.chambersmd@gmail.com

Received: January 17, 2019; Accepted: January 22, 2019; Published: January 25, 2019 